not surprising that they are often the first witness.

- Parents who are unusually calm for the severity of illness - Parents who appear outwardly calm may be suffering inner turmoil.

- Parents who are unusually knowledgeable about the illness and its repercussions - This is common in concerned parents and those with a chronically ill child, particularly if they have been properly informed by the doctors.

- Parents who fit in contentedly with ward life and attention from staff-This is common when the children have been in a ward several times or where the ward is well organised and the staff caring and compassionate.

- Unusual or unexplained illness or death in previous children - It is important not to jump to conclusions but to investigate the details of the deaths or illnesses.

- Parents who have a history of unusual illness or themselves were abused as children - This needs careful history taking, scrutiny of the notes, or confidential discussion with the mother's doctors.

- Parents who have a history of conduct or eating disorders - These are relatively common in the community. Most of these people do not abuse their children.

The accuracy and predictive value of these diagnostic pointers are not known. As Munchausen syndrome by proxy is very rare these pointers are much more likely to be associated with normal behaviour and common illnesses. Unthinking use of such indicators without realising their non-specific nature may lead to mothers being falsely accused and their children taken into care.

\section{Concern about 'exaggeration' being} labelled Munchausen syndrome by proxy Munchausen syndrome by proxy is being used to describe the behaviour of mothers who exaggerate a child's symptoms. This is a cause for concern because mothers frequently exaggerate their child's symptoms, not through any malignant desire to mislead the doctor but as part of common language: 'he hasn't eaten a thing all week', 'he vomits up all the feed'. Such phrases are part of everyday life and experienced paediatricians do not take the mother's story at face value but take a careful history to find out exactly what has been happening. We have no idea how common it is for mothers to exaggerate their children's symptoms. If exaggeration is included in Munchausen syndrome by proxy this will devalue the diagnosis and more mothers will be accused.

\section{Concern about inadequate history taking}

Diagnosing Munchausen syndrome by proxy is very serious because the outcome for the child and family may mean care orders, separation, and even criminal prosecution. It is primarily a medical diagnosis and must be based on sound medical practice. This means taking a very careful history, eliciting exact details about what has happened. ${ }^{4}$ It may mean talking to the mother for hours to get a clear understanding about all the episodes, why she is concerned, and her own background. Unfortunately, in some cases, the senior doctors have hardly talked to the mother. In a recent case the mother briefly saw the consultant during the children's illnesses and he did not take time to sit down and talk with her until he came with a social worker to say she was accused of Munchausen syndrome by proxy. This is indefensible.

My experience is that some mothers repeatedly take their child to the family doctor or paediatrician because they are anxious. Some are naturally anxious; some have hypochrondia; and others are anxious because their children have previously been very ill. The doctor realises the illnesses are trivial and repeatedly tells the mother not to worry. If the doctor had taken the time, in the early part of the illness, to take a full history, to explain what was happening, understand why she was so concerned, and support the mother, he could have prevented many of the repeated presentations and the subsequent accusation of Munchausen syndrome by proxy.

\section{In conclusion}

Munchausen syndrome by proxy is a term I suggest we use with caution and preferably abandon in favour of giving an exact description of what has happened to the child. It has become a diagnosis with emotional overtones which do not help in the management of these difficult cases. I do not consider that a mother exaggerating genuine symptoms or signs should be considered part of Munchausen syndrome by proxy. I urge caution about some of the criteria for this diagnosis and concern about some to the accuracy, sensitivity, and ethics of some techniques being used. I urge doctors to take detailed histories and talk to the mothers in a caring way about their concerns. It is important to protect a child who is being harmed by his mother. It is equally important not to harm the child by falsely accusing his mother of Munchausen syndrome by proxy thereby breaking up the family.

\section{The Allitt inquiry. London: HMSO, 1994. \\ 2 Meadow SR. 'Who's to blame - mothers, Munchausen or medicine?' $f R$ Coll Physicians Lond 1994; 28: 332-7. \\ 3 Samuels MP, Southall DP. Munchausen syndrome by proxy. Br f Hosp Med 1992; 47: 759-62. \\ 4 Working Party of the British Paediatric Association. Evaluation of suspected imposed upper airway obstruction. London: BPA, February 1994: 24 (section 6.2.5).}

\section{Commentary}

The concerns which Dr Morley expresses over the diagnosis of Munchausen syndrome by proxy are numerous but fall into two main area. These are the appropriateness of the label and the specificity of the criteria used in the diagnosis.

THE LABEL

There is no doubt, despite claims in the press, that Beverley Allitt did not have Munchausen 
syndrome by proxy. The main feature of the syndrome is that 'the affected person invents or creates symptoms in his or her charge in order to gain medical attention'. ${ }^{1}$ There is no evidence to suggest that this was ever Miss Allitt's motive. I remain convinced that it is a useful diagnostic term and that it is not invalidated by its misuse on a single occasion.

\section{SPECIFICITY}

Dr Morley is right that many of the criteria used to identify situations in which Munchausen syndrome by proxy should be suspected are relatively non-specific and cannot be considered as diagnostic on their own. Nevertheless the presence of several criteria does, if nothing else, raise the chances that the child is at risk. All paediatricians would obviously agree that it is crucial to listen carefully to the information provided by the carers and I am sure we would all also agree that at times we fail in this respect.

Although it is obviously true that the child will probably spend more time with the carer under suspicion than other family friends and relatives, natural recurrent events usually are witnessed by others at some time or another. It is therefore essential to obtain independent corroborative evidence rather than relying on the statement of the carer. It is also important to stress that the onset of the event must be seen as otherwise the cause will remain in doubt. $^{2}$
Inconsistent histories are a more useful pointer than has been claimed by Dr Morley. Much weight has been placed on the importance of identifying inconsistencies in the description of events in the diagnosis of child abuse. If they are helpful in that condition they are also useful in the identification of Munchausen syndrome by proxy. It is possible to make a case that the only diagnostic role of overt cardiorespiratory (or electroencephalographic) ${ }^{3}$ monitoring is that a sequence of events can be documented which may differ very considerably from the history described by the carer.

Sadly, along with child physical and sexual abuse Munchausen syndrome by proxy does occur and can constitute a real threat to the survival of the child. Inevitably if we are to identify these children, we will have to suspect some carers who are totally innocent. Dr Morley's article reminds us of the effects of these suspicions on the carer and the family. We cannot, however, put our heads in the sand and pretend that Munchausen by proxy does not occur.

A D MILNER Department of Paediatrics, United Medical and Dental School St Thomas's Hospital, London SE1 7EH

1 Meadow SR. Munchausen syndrome by proxy. The hinterland of child abuse. Lancet 1977; ii: 343-5.

2 Meadow SR. 'Who's to blame - mothers, Munchausen or medicine?' $7 R$ Coll Physicians Lond 1994; 28: 332-7.

3 Stephenson JPB. Video surveillance in diagnosis of intended suffocation. Lancet 1994; 344: 414-5.

\title{
Is Munchausen syndrome by proxy really a syndrome?
}

\author{
Geoffrey C Fisher, Ian Mitchell
}

Faculty of Medicine, University of Calgary, Departments of Psychiatry and

Paediatrics G C Fisher

Departments of

Paediatrics

I Mitchell

Correspondence to: Dr G C Fisher, University of Calgary, Alberta Children's Hospital, 1820 Richmond Road SW, Calgary, Alberta T2T 5C7, Canada.
Munchausen syndrome by proxy was first described by Meadow in $1977 .{ }^{1}$ Given the trend against the use of eponyms it has been suggested that a more appropriate term is factitious illness by proxy. ${ }^{2}$ We have reviewed the syndrome elsewhere $^{3}$ and essential criteria for diagnosis have been provided by Rosenberg. ${ }^{4}$ Meadow has advanced a useful list of warning signs. ${ }^{5}$ Munchausen syndrome by proxy/factitious illness by proxy is thought to be rare. A parent, nearly always the mother, falsifies illness in her child or children by fabricating a history and/or by producing symptoms or signs. The child is presented for medical care with 'illnesses' that are unexplained, prolonged, and unresponsive to all approaches. Symptoms occur only in the mother's presence.

Almost any clinical picture can be fabricated. ${ }^{4-8}$ Common presentations are epileptic seizures $^{69}$ and infant apnoea, ${ }^{10-12}$ though fabrications of complex or rare diseases occur. ${ }^{13-17}$ Munchausen syndrome by proxy/ factitious illness by proxy is the cause of significant morbidity and mortality. Sometimes more than one child in the same family is victimised. ${ }^{18-20}$

The mothers have unusually close relationships with hospital staff, and about one third have previous complete or partial nurse training or other health profession associations. Many varied psychiatric diagnoses have been applied to the perpetrators, and some demonstrate features of Munchausen's syndrome themselves, though how many is uncertain. Distant and uninvolved partners are typical and the mothers appear to have few social outlets. We can call these prototypical cases.

\section{'Not quite Munchausen syndrome by proxy/factitious illness by proxy'}

Physicians are well aware of parents who anxiously insist that their children are ill. In paediatric practice the parent who claims their child has 'multiple allergies' is common, as is the overprotection of epileptic children because of suspected seizures. In some cases, as a result of parental anxieties, severe restrictions 